\title{
New Algorithms for Energy-Efficient VLC Networks with User-Centric Cell Formation
}

\author{
Mohanad Obeed, Student Member, IEEE, Anas M. Salhab, Senior Member, IEEE, Salam A. Zummo, Senior \\ Member, IEEE, and Mohamed-Slim Alouini, Fellow, IEEE
}

\begin{abstract}
The ever-increasing demand in high data rate has pushed the attention of the researchers to utilize the unregulated visible light spectrum for communication. This paper proposes a joint access-point (AP) association and power allocation algorithms for energy-efficiency (EE) maximization in visible light communication (VLC) networks. Based on the user-centric (UC) design, we first show that the cell formation and power allocation are interlinked problems and should be treated jointly. We start by proposing a new algorithm for users' clustering and then associating all the APs to the clustered users based on a proposed metric. We then propose two algorithms that jointly allocate the power, under quality-of-service $(Q 0 S)$ constraints, and decide which APs must be prevented from participating in communication. The first algorithm is designed to maximize the EE, while the other algorithm is designed to reduce the complexity of the first algorithm with acceptable degradation in the EE. Different from the related literature that allocated the power with the worst case interference information, we propose an iterative algorithm that allocates the power based on exact interference information, which significantly improves the EE. The numerical results demonstrate that the proposed algorithms significantly improve the EE compared to the existing work.
\end{abstract}

Index Terms-Visible light communication, energy efficiency, user clustering, power allocation.

\section{INTRODUCTION}

The growing demand for high data rate and licencefree spectrum applications has stimulated recently many researchers to investigate the visible light communication (VLC) as a promising technique for indoor communication. As known, the radio frequency (RF) communications are becoming more restricted because of the limited spectrum resources in wireless networks. As a solution, the VLC has been introduced in indoor environment to overcome the RF limitations and provide better services to the users.

VLC is a communication technology that uses light emitting diode (LED), which is highly energy efficient, as a transmitter to emit both the light and information signals to the users. It is important to note that the input current to LEDs must be in the range of illumination requirement and in the range of the LEDs' physical limits. The receiver must be equipped with a photo detector (PD) device that converts optical signals to electrical signals. The data is transmitted, using an intensity

Part of this work has been presented in IEEE ICC'2018 [1].

M. Obeed, A. M. Salhab, and S. A. Zummo are with the King Fahd University of Petroleum \& Minerals (KFUPM), Dhahran, Eastern Province, Saudi Arabia (email: g201106250@kfupm.edu.sa, salhab@kfupm.edu.sa, zummo@kfupm.edu.sa).

M.S. Alouini is with the King Abdullah University of Science and Technology (KAUST), Thuwal, Makkah Province, Saudi Arabia (email: slim.alouini@kaust.edu.sa) modulation (IM) technique at the transmitter, and received, using a direct detection (DD) technique at the receiver (IM/DD). Unlike RF networks, VLC technology works properly only when the line-of-sight (LoS) component between transmitters and receivers is available. For instance, the opaque placed in the indoor environment might block the LoS light that is carrying the data for some intended users which leads to degrade the channel up to 90 percent of the LoS channel [2] and the data rates at intended users would be deteriorated significantly.

VLC networks has been considered as an energy-efficient technology [3], as the LEDs, which are used as transmitters, are energy-efficient devices [4], and because the consumed power used for communication is also used for illumination. However, the acceptable illumination is ranging between maximum and minimum requirements, which means that the consumed power can be controlled within these limits to maximize the EE. In other words, the extra power consumed for communication should be minimized with keeping the required quality of service (QoS) achieved to improve the system's EE. In addition, with the advent of $5 \mathrm{G}$ of wireless networks, the tremendous number of access points (APs) and billions of connected devices, the demand for designing energy-efficient systems is becoming even more compelling for seeking to have green communication systems [3]. This is confirmed, numerically, by what is shown in this paper (in the simulation part) that the EE in VLC networks is highly negatively affected by increasing the number of active users.

Extensive work has been done for throughput and system capacity maximization in VLC networks using different modulation schemes [5], [6], power allocation allocation and load balancing [7]-[12], and using multi-input single-output (MISO) [13] and multi-input multi-output (MIMO) schemes [14]. Cooperation between APs has also been proposed to enhance the VLC system performance by mitigating the interference [15], [16], and decreasing the handover with mitigating the blockages effect [17].

Despite all the aforementioned advances, few papers in the literature addressed the design and optimization issues of the energy efficiency (EE) in VLC networks. Aiming to maximize the EE in VLC, several factors have been employed in the literature such as cell formation, power allocation, and link level optimization. Because the inter-cell interference is a major reason behind the quality-of-service (QoS) and $\mathrm{EE}$ degradation, the cell formation has been proposed to mitigate this problem by grouping multiple APs to be in a one cell. Efficient cell formation design is a crucial step that has a 
great impact on the EE of the system. In a highly dense VLC networks, when the number of users is much less than the number of APs, the user-centric (UC) design for VLC networks is an appropriate technique for cell formation. In [18], the authors studied the UC clustering for VLC, where the formed cells structure do not have a specific shape. They first clustered the users then associated the APs to the grouped users. Authors of [19] expanded the work to consider the fairness among users and proposed algorithms for scheduling the users to maximize the sum utility of the proposed system. Authors of [20] allocated the powers for the clustered users aiming to maximize the $\mathrm{EE}$ of the distributed cells.

All the previous works [19]-[21] studied the system level optimization for energy efficient VLC systems by solving the cell formation and power allocation separately, while they are interlinked problems. Besides, previously, the power allocation optimization problem was solved under the assumption that the interference information at each user is at its maximum value (inaccurate interference information), resulting inefficient power allocation schemes. Finding the global optimal solution for the joint cell formation and power allocation is an extremely difficult problem. However, we propose a sub-optimal solution that considers both the APs association, which is a part of the cell formation, and power allocation jointly. In other words, to simplify the problem, we implement the users' clustering procedure independently (with considering the inter-cell interference), and implement the other two procedures (APs selection and power allocation) jointly.

In this paper, we design an energy-efficient VLC network by proposing new efficient algorithms that jointly form the cells, allocate the power for the users, and select the appropriate APs. More specifically,

- We propose a new user clustering algorithm that aims at minimizing the distance of the clustered users to their centers and maximizing the distance between the different centers in order to mitigate the inter-cell interference.

- We establish a metric for each AP in order to select the appropriate users' cluster to work on.

- We show that the power allocation and APs association problems are not independent as tackled in the previous works. Therefore, we develop a new algorithm that finds a solution for joint power allocation and AP selection, resulting in a significant improvement in the EE.

- We propose a low-complexity solution for the joint problem of power allocation and APs selection aimed at decreasing the complexity with an acceptable degradation in the EE compared to the first proposed algorithm.

- In the power allocation subproblem, in the previous works [19]-[21], authors allocated the power based on the worst case of inter-cell interference which is a fake interference information. Here, we propose an iterative algorithm that approaches to having the exact interference information and improves the $\mathrm{EE}$ as the number of iterations increases. In addition, for solving the power allocation problem, we modify the traditional subgradient method by introducing closed-form expression for some dual variables, resulting in speeding up the convergence.

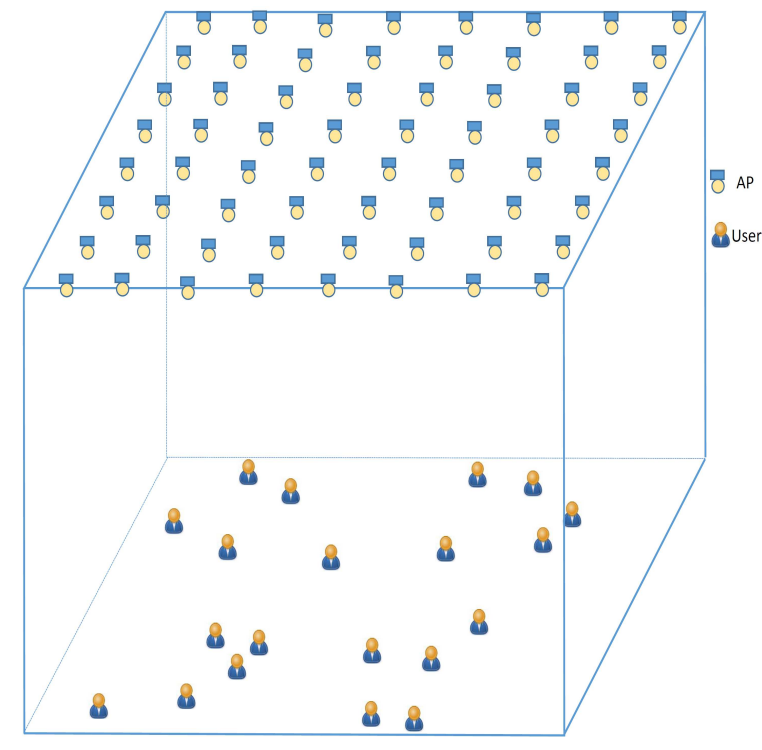

Fig. 1. System model (an example of distributing the users uniformly in the area).

The rest of this paper is organized as follows. The system and channel models are introduced in Section II. In Section III, we present the problem formulation and the proposed solutions. Some simulation results are presented and discussed in Section IV. Finally, the paper is concluded in Section V.

\section{System ANd Channel Models}

The system under consideration consists of $N_{A}$ VLC APs and $N_{u}$ users, as shown in Fig. 1. The users are distributed uniformly in the area and the APs are fixed in the ceiling of the room. Each AP is equipped with multiple LEDs that use IM to transmit the light signal to the users that can receive convert the light to current, using PDs. Also, the locations of users are assumed to be unchanged during a short time duration $T$. This assumption can be justified by assuming that all the mobile users are better to be served by WiFi APs to avoid the numerous handover usually occurs in VLC networks [9], [22]. Thus, the channel-state-information (CSI) of the VLC links is considered to be constant during $T$ period. Several indoor environments can prove that the number of lamps fixed in the ceiling are more than the number of users inside the considered room such as labs, houses, offices, companies, etc. This motivates us to assume that the number of users is less than the number of APs. However, the proposed algorithms in this paper can be implemented even if the number of users are more than the number of APs. For instance, if the number of users are twice the number of APs, a user scheduling algorithm can be implemented where half of the users served by the first time slot while the others served in the second time slot, and the proposed procedures can be implemented within each time slot.

\section{A. Channel Model}

The receiver receives the LoS optical signal beside many copies of it coming from reflections. According to [23], the 
optical power received from all the more than once reflected links are negligible. The line-of-sight (LoS) VLC channel between the $i^{t h}$ LED and the $j^{t h}$ user can be expressed as follows [24]

$$
h_{i, j}^{(L o S)}=\frac{(m+1) A_{p}}{2 \pi d_{i, j}^{2}} \cos ^{m}(\phi) g_{o f} f(\theta) \cos (\theta),
$$

where $m$ is the Lambertian index that is given by $m=$ $-1 / \log _{2}\left(\cos \left(\theta_{1 / 2}\right)\right.$, where $\theta_{1 / 2}$ is the half intensity radiation angle, $A_{p}$ is the physical area of the PD, $d_{i, j}$ is the distance between the $i^{t h}$ LED and the $j^{t h}$ user, $g_{o f}$ is the gain of the optical filter, $\phi$ is the angle of radiance at the LED, $\theta$ is the angle of incidence at the $\mathrm{PD}$, and $f(\theta)$ is the optical concentrator gain, which is a function of $\theta$ that is given by $f(\theta)=\frac{n^{2}}{\sin ^{2}(\Theta)}$ if $\theta \leq \Theta$ or 0 if $\theta>\Theta$, where $n$ is the refractive index and $\Theta$ is the semi-angle of the field-of-view (FoV) of the PD. Authors of [23] showed that the channel's DC attenuation from the first reflected link is given by

$$
\begin{aligned}
h_{i, j}^{N L o S}= & \frac{(m+1) A_{p}}{2 \pi d_{k, i}^{2} d_{j, k}^{2}} \cdot \rho \cdot d A_{s} \cdot \cos ^{m}\left(\phi_{r}\right) \cdot \cos \left(\alpha_{1}\right) \cdot \cos \left(\alpha_{2}\right) \\
& . g_{o f} f\left(\theta_{r}\right) \cdot \cos \left(\theta_{r}\right),
\end{aligned}
$$

where $\phi_{r}$ and $\theta_{r}$ are the angels of the irradiance and incidence of the first reflection link, respectively, $d_{k, i}^{2}$ and $d_{j, k}^{2}$ are the distance from the AP $i$ to the reflecting point $k$ and the distance from the reflecting point $k$ to the user $j$, respectively, $\rho$ and $d A_{s}$ are the reflection factor and the reflective area, respectively, $\alpha_{1}$ and $\alpha_{2}$ are the the irradiance angles with respect to the reflected point and with respect to the receiver, respectively. Hence, the equivalent VLC channel between AP $i$ and user $j$ is given by:

$$
h_{i, j}^{(v)}=h_{i, j}^{(L o S)}+h_{i, j}^{N L o S} .
$$

\section{B. Transmission Technique}

For white LED transmission, we use the single blue LED chip with a phosphor layer, which is commonly used, where the modulation bandwidth $B$ is normally around $20 \mathrm{MHz}$, however this measured bandwidth is related to the specific LED product employed. The energy-efficient asymmetrically clipped optical OFDM (ACO-OFDM) is used as a modulation scheme in the proposed system model. In each formed $c$ th cell, we have $N_{A, c}$ APs transmitting the signal vector $\mathbf{Y}_{t, c} \in \mathbb{R}^{N_{A, c} \times 1}$ to $N_{u, c}$ users. The equivalent channel can be modeled as a multi-users MISO system and the vectored transmission (VT) zero-forcing based is used to eliminate the intra-cell interference. Hence, we can express the received signal vector of a particular sub-channel in the cluster $c$ by

$$
\mathbf{Y}_{r, c}=\rho \mathbf{H}_{c} \mathbf{G}_{c} \overline{\mathbf{P}}_{c} \mathbf{Y}_{t, c}+\mathbf{n}_{\mathbf{c}}
$$

where $\mathbf{H}_{c} \in \mathbb{R}^{N_{u, c} \times N_{A, c}}$ is the channel attenuation matrix between the end users and the APs in the cluster $c$, $\overline{\mathbf{P}}_{c}=\operatorname{diag}\left(\mathbf{P}_{c}\right)$, where $\mathbf{P}_{c}$ is the electronic power vector assigned to the users belong to the cluster $c, \mathbf{n}_{\mathbf{c}}$ is the noise plus the inter-cell interference, and $\mathbf{G}_{c}$ is the pre-coding matrix that is designed to diagonalize the channel matrix by setting
$\mathbf{G}_{c}=\mathbf{H}_{\mathbf{c}}{ }^{H}\left(\mathbf{H}_{\mathbf{c}} \mathbf{H}_{\mathbf{c}}{ }^{H}\right)^{-1}$. This process (diagonalizing the channel or eliminating the intra-cell interference) needs the accurate knowledge of the users' channels. Since the VLC channels are pre-dominantly static, the user's channel can be attained using a single attenuation factor. It can be estimated at the user side and then fed back to the AP side at the cost of modest overhead [20]. It is important to note that the amount of the exchanged information depends on the cluster size or the number of cells in the VLC systems. A low number of cells (large cells' size in average) leads to a less inter-cell interference and a large amount of exchanged information, while a high number of cells (small cells' size in average) leads to a high inter-cell interference and a small amount of exchanged information. Hence, the number of cells must be selected carefully based on the needed applications. The achievable data rate at the user $j$ in cluster $c$ is given by

$$
R_{j, c}=\beta \log \left(1+\frac{\left(\rho^{2} / 2\right) P_{j, c}}{B N_{0}+X_{c, j}}\right),
$$

where $P_{j, c}$ is the assigned power for the user $j$ in the cell $c, X_{c, j}$ is the interference received at the user $j$ in the cell $c, N_{0}$ is the noise power spectral density, and $\beta$ is a value which depends on the applied modulation scheme. Because of the assumption that the (ACO-OFDM) modulation scheme is applied, the value of $\beta=B / 4$, where $B$ is the modulation bandwidth. The transmit power at AP $i$ in the cluster $c$, which is the power consumed for communication, is given by

$$
p_{i, c}=\sum_{j=1}^{N_{u, c}} g_{i, j}^{2} P_{j, c},
$$

where $g_{i, j}$ is the element located in the $i^{\text {th }}$ row and $j^{\text {th }}$ column in matrix $\mathbf{G}_{c}$.

\section{Problem Formulation}

In this section, we present algorithms and steps to efficiently form the cells and allocate the powers to maximize the EE. The general procedures are represented in Fig. 2, where the difference between the proposed and the traditional procedures [19]-[21] is shown. We start by proposing a users' clustering algorithm, establishing metrics for associating all APs to the clustered users, formulating the power allocation optimization problem with providing an efficient solution, and proposing two different iterative algorithm (one for seeking the optimality and the other for seeking the simplicity) to jointly allocate the power and select the participating APs in communication. These individual procedures are followed by a flowchart that shows how the proposed algorithms and steps are arranged and implemented, in general, to maximize the EE.

\section{A. Cell Formation}

Here, we provide a new UC clustering algorithm under a given number of clusters, we then provide a new procedures for APs association to the formed users' clusters. Our targets in users' clustering are to cluster the users based on their distances to each others and to maximize the separation between the clusters. The main contribution of our clustering 


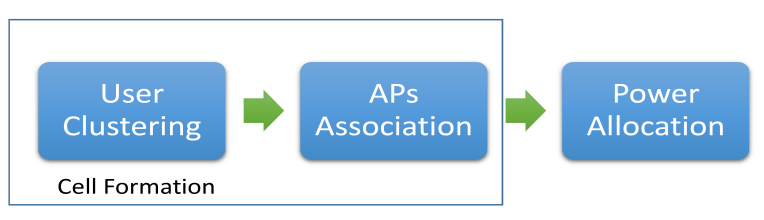

(a) Traditional procedures.

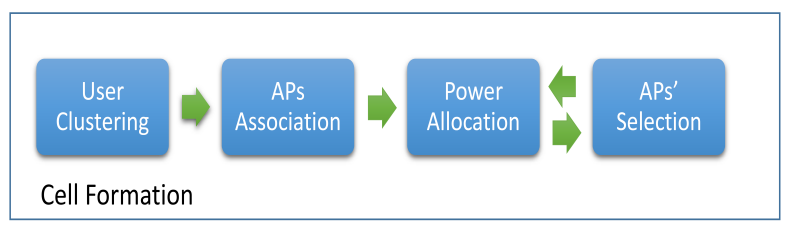

(b) Proposed procedures.

Fig. 2. Comparison between the proposed and the traditional procedures for EE maximization.

approach is the initial steps that have a significant impact on the final results in terms of EE.

1) User-centric clustering: In this section, we aim to group the users in a predefined $K$ clusters aiming to maximize the EE of the whole network. Clustering the users based on their geographical positions, to have the summation of the distances of all users in a cluster to their centers as small as possible, definitely helps in improving the EE of the system. Due to its simplicity and speed, The traditional K-means clustering method has been widely used in clustering. It is designed to minimize the following objective function

$$
U=\sum_{k \in K} \sum_{j \in c}\left\|\mathbf{x}_{j, k}-\mathbf{c}_{k}\right\|^{2},
$$

where $\mathbf{x}_{j, k}, \mathbf{c}_{k} \in \mathcal{R}^{2}$ are the position of the user $j$ in the cluster $k$ and the center position of the cluster $k$, respectively. There are multiple improved versions of the K-means method in the literature. Here, we pick the improved version of the Kmeans method proposed in [25], which is called $\mathrm{K}$-means++, to build on. Briefly, the K-means++ augmented the K-means method with a randomized seeding technique which helps in improving the speed and the accuracy. More details on the $\mathrm{K}-\mathrm{mean++}$ clustering method are provided in [25].

If a different clustering schemes are given, it is highly complex to implement both the APs association and power allocation to specify the best clustering scheme that provides the highest EE. To simplify the problem, first several clustering design are offered, then the best clustering design that helps in mitigating the inter-cell interference is estimated. The main disadvantage in the $\mathrm{K}$-means++ method is that the selection of the initial centers may lead to a poor EE. This is because it is based on a probabilistic initial selection and it is not built for the special structure of the VLC networks that is highly affected by the inter-cell interference. Therefore, beside the objective of the K-means++ algorithm, we augment another objective that helps in decreasing the inter-cell interference. This objective is to maximize the average distance between the users in the different clusters. This means having the distances between the cluster centers as far as possible. To achieve such objective, it is important to note that if $K$ points are given and the distances between them $d_{i, j}, i=1, \ldots, K, j=$ $i+1, \ldots, K$, under the constraint that the summation of them is less than or equal $D$, the solution of the following optimization problem

$$
\max _{d_{i, j}} \prod_{i=1, j=i+1}^{K} d_{i, j}, \quad \text { s.t. } \sum_{i=1, j=i+1}^{K} d_{i, j} \leq D,
$$

is $d_{i, j}=\frac{D}{K}, i=1, \ldots, K, j=i+1, \ldots, K$. This means that if we have $M K$ centers, the $K$ centers out of $M K$, which have maximum distance between them, can be found by finding the product of the distances of all possible $K$ centers and picking up the maximum result, where $M$ is an integer value and greater than or equal 1 .

The main idea of the proposed algorithm is to start with a number of initial centers that is much more than $K$, then select the centers that have the maximum distance between them. Therefore, the following Algorithm 1 is the proposed clustering algorithm.

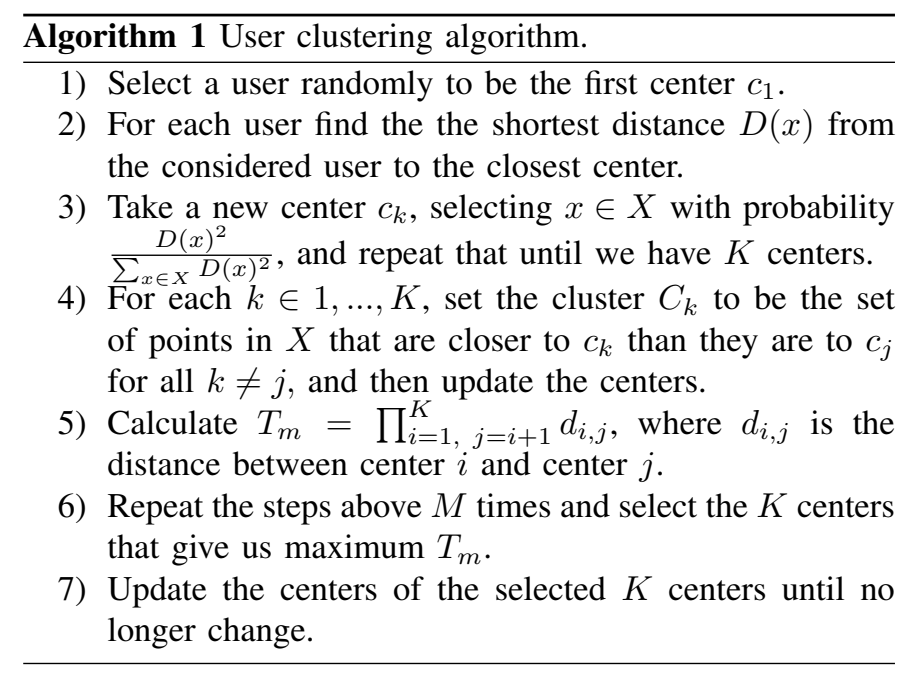

Increasing $M$ would enhance the performance and increase the complexity, and decreasing it would decrease the complexity and degrade the performance. The complexity of Algorithm 1 is at most $M$ times the complexity of the $\mathrm{K}$-means++ algorithm, but it yields a high impact on the EE, and it is negligible if we compare it with the complexity of the power allocation problem. It is important to note that the proposed clustering algorithm requires the location of all users to be known. The users' locations depend on the channel knowledge at APs. Once the APs acquire the users' channels, the distances between users can be inferred using the equation of the channel model (1) [20]. Then, classical positioning and tracking can be used to determine the users' locations [26]. As a result, the users' locations would be known at AP side, where the clustering method can be implemented.

2) AP Association: It is plausible that increasing the number of APs in a cluster improves the EE of that cluster, but on the other hand, it increases the inter-cell interference in the other clusters. This inspires us to propose a new cell formation technique that first involves all the APs in the network, then switches off the ones that are harmful (in terms of increasing 
the inter-cell interference) more than helpful (in terms of enhancing the EE inside the cell). Here, we distribute the APs to the clustered users, and in Section III-C, we optimize the APs selection based on a given power allocation scenario. Our goal here is that for each AP, we select the best cluster that might serve the associated users or enhance the EE as possible as it can. Therefore, we propose two steps to associate the APs to the formed cells:

1) We assign for each user its closest AP by using the following steps: 1) In the channel matrix, find the maximum channel value, assign the corresponding AP to the corresponding user, then make the row and the column of the corresponding pair equal to zero, 2) Repeat Step (1) until the channel matrix equals the zero matrix.

2) For the remaining APs, for each cluster $c$, we first find the average channel of each AP to all users in the cluster $c$ as follows

$$
\hat{g}_{i, c}=\sum_{j \in c} h_{j, i}^{2} \cdot c=1, \ldots, K, i=1, \ldots, N_{A} .
$$

Then, we associate the AP $i$ to the cluster that satisfies the following relation

$$
I_{i}=\max _{c}\left(\hat{g}_{i, c}\right), c=1, \ldots, K .
$$

The target of the second step is to associate the AP to the cluster that would maximize the cell capacity and minimize the inter-cell interference in the other cells.

In Fig. 3, we illustrate the cell formation by showing an example of clustering a given distributed users to a three clusters using our proposed Algorithm 1 in Fig. 3a. In addition, we show how the proposed steps in the APs association associate the APs to the clustered users in Fig. 3b. Fig. 3b shows that the number of APs associated to cluster $c$ is directly proportional to the the number of the users in that cluster.

\section{B. Power Allocation Scheme}

Our goal in this section is how to allocate the power efficiently for the $N_{u}$ users to maximize the EE. Specifically, the objective function is to maximize the EE of the whole network under certain QoS constraints and maximum available power constraints. These constraints are introduced to guarantee some fairness among users per cell and to achieve the required illumination, respectively. The EE function is defined as the ratio of benifit provided by the system over the total consumed power [3]. In other words, if the system benefit is the sum rate, hence the energy efficiency is given by:

$$
F_{E E}=\frac{R_{T}}{P_{T}}
$$

where $R_{T}$ is the sum rate, $P_{T}$ is the total consumed power at the transmitters. In this paper, we consider only the consumed power for communication in the formulated EE function. In the literature, two types of EE function have been introduced, which are the average per-cell EE and the global EE (GEE). For the average per-cell EE, let us define $\eta_{c}$ as the EE at the cell $c$, hence, using Equations (5) and (6), the average per cell $\mathrm{EE}$ of the whole network is defined as

$$
E E=\frac{1}{K} \sum_{c=1}^{K} \eta_{c}
$$

where

$$
\eta_{c}=\frac{\beta \sum_{j=1}^{N_{u, c}} \log \left(1+\frac{\left(\rho^{2} / 2\right) P_{j, c}}{B N_{0}+X_{c, j}}\right)}{\sum_{i=1}^{N_{A, c}} \sum_{j=1}^{N_{u, c}} g_{i, j}^{2} P_{j, c}} .
$$

The global EE (GEE) is the summation of data rates of users in all clusters over the total power consumed at all APs, which can be expressed as

$$
G E E=\frac{\beta \sum_{c=1}^{K} \sum_{j=1}^{N_{u, c}} \log \left(1+\frac{\left(\rho^{2} / 2\right) P_{j, c}}{B N_{0}+X_{c, j}}\right)}{\sum_{c=1}^{K} \sum_{i=1}^{N_{A, c}} \sum_{j=1}^{N_{u, c}} g_{i, j}^{2} P_{j, c}} .
$$

Function (13) is usually optimized when the centralized approach is employed, while Function (11) supports an efficient distributed approach. We select to optimize the average per-cell EE for its simplicity and for the reason that it can be implemented in each cell independently. The optimization problem can be formulated as follows:

$$
\begin{array}{cl}
\max _{P_{j, c}, \forall j, c} & E E, \\
\text { s.t. } & R_{j, c} \geq \gamma, \forall j, c, \\
& \sum_{j=1}^{N_{u, c}} g_{i, j}^{2} P_{j, c} \leq p_{\max }, \forall j, c, \\
& P_{j, c} \geq 0, \quad \forall j, c,
\end{array}
$$

where $\gamma$ is the minimum required data rate, and $p_{\max }$ is the maximum electronic transmit power available at the APs. It is important to note that the $p_{\max }$ must be selected to satisfy the illumination requirements and must be within the operational dynamic range of the LEDs. A detailed discussion about optimizing the value of $p_{\max }$ is provided in [20]. Problem (14) can be separated equivalently to $K$ subproblems, where the optimization problem in cluster $c$ can be expressed as follows:

$$
\begin{array}{cl}
\max _{P_{j, c}, j=1, \ldots, N_{u, c}} & \eta_{c}, \\
\text { s.t. } & R_{j, c} \geq \gamma, j=1, \ldots, N_{u, c}, \\
& \sum_{j=1}^{N_{u, c}} g_{i, j}^{2} P_{j, c} \leq p_{\max }, \\
& i=1, \ldots, N_{A, c}, \\
& P_{j} \geq 0, \quad j=1, \ldots, N_{u, c} .
\end{array}
$$

Problem (15) must be implemented in each cell independently aiming to maximize the summation of the cells' EE in the whole network. Problem (15) is not easy to be solved because the inter-cell interference terms depend on the allocated powers and the power allocation problem depends on the interference terms. In order to simplify this problem, we first solve it under the worst case where the interference is considered to be at its highest value, which happens when all the APs are assumed to transmit at their maximum power. Then in Section III-B1, we provide an algorithm that solves 


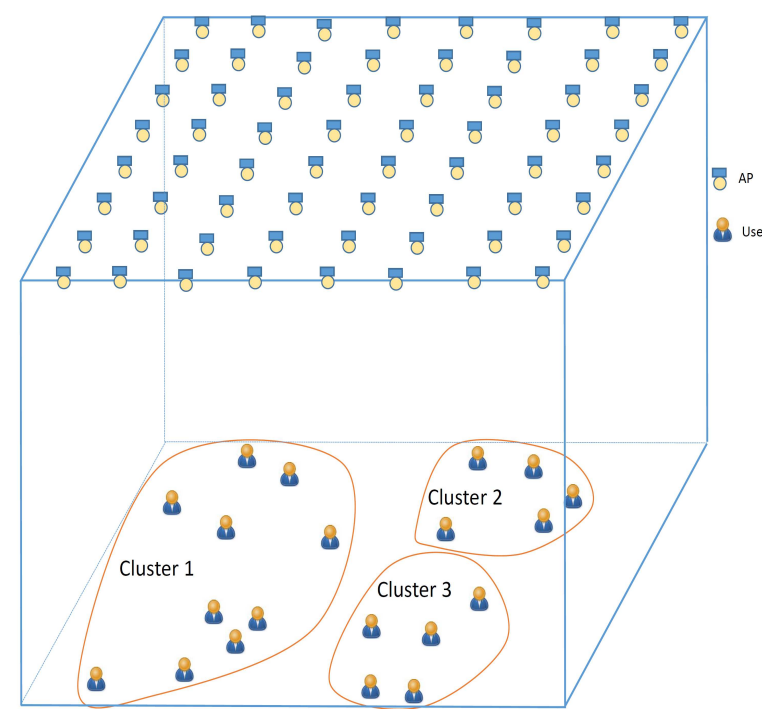

(a) User clustering.

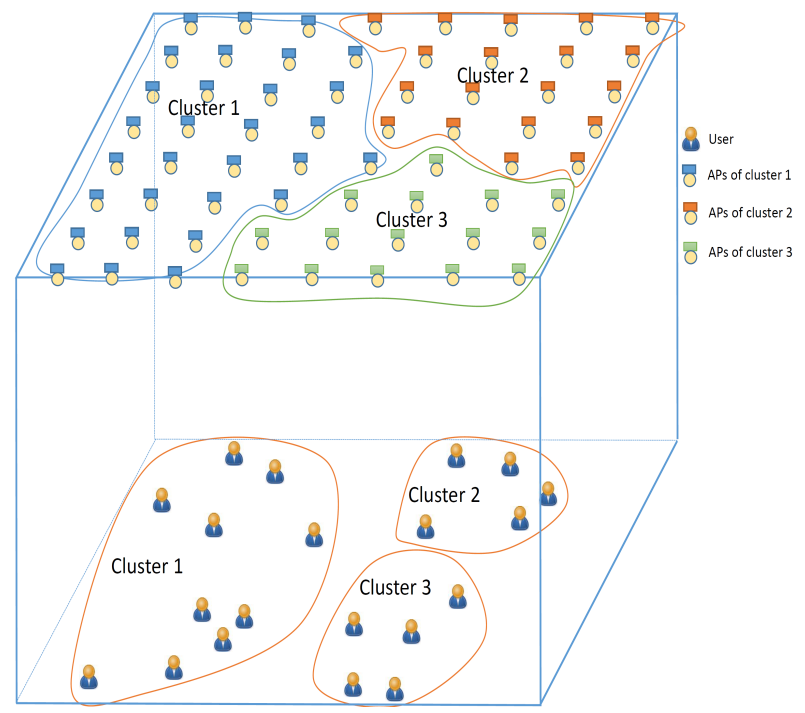

(b) Associating APs.

Fig. 3. An example of cell formation a) User clustering using Algorithm 1, b) Associating APs using the proposed steps, when $K=3$ and $\mathrm{H}-\mathrm{FoV}=60^{\circ}$.

the power allocation problem based on the exact or the approximate interference information. Even if we assume the interference information is available, Problem (15) is still not easy to solve since the objective function in (15) is a ratio of two functions (concave in the numerator and a linear function in the denominator), which is generally considered as a nonconvex function. It is noted that there is no standard approach for solving non-convex optimization problems. However, this class of optimization problems, can be solved using fractional programming tools [27], [28] such as the Dinkelbach method [29] that transforms this problem into a successive concave functions. In the following, we decompose the solution of (15) into two loops:

Main Loop: Using Dinkelbach method, we can transform the objective function in (15) to be as

$$
\begin{array}{ll}
\max _{P_{j, c}, j=1, \ldots, N_{u, c}} & \beta \sum_{j=1}^{N_{u, c}} \log \left(1+\frac{\left(\rho^{2} / 2\right) P_{j, c}}{B N_{0}+X_{c, j}}\right) \\
& -q_{c} \sum_{i=1}^{N_{A, c}} \sum_{j=1}^{N_{u, c}} g_{i, j}^{2} P_{j, c}, \\
\text { s.t. } & (15 a),(15 b),(15 c),
\end{array}
$$

where $q_{c}$ is a positive variable introduced to be optimized or selected to have the objective function in (16) equal to zero at optimality. If $q_{c}$ is given, Problem (16) is concave and can be solved using the classic dual-decomposition method, since Slaters' conditions are satisfied [30], which means that the duality gap is zero. In order to find the optimal $q_{c}$, the Dinkelbach algorithm is used to find the optimal value of $q_{c}$ by the following steps: Step 1) Put $q_{c}=0$ as an initial value, Step 2) Solve Problem (16) using the approach provided in the inner loop, and let $P_{j, c}^{*}$ as the optimal solution, Step 3) update $q_{c}$ by

$$
q_{c}=\frac{\beta \sum_{j=1}^{N_{u, c}} \log \left(1+\frac{\left(\rho^{2} / 2\right) P_{j, c}^{*}}{B N_{0}+X_{c, j}}\right)}{\sum_{i=1}^{N_{A, c}} \sum_{j=1}^{N_{u, c}} g_{i, j}^{2} P_{j, c}^{*}} .
$$

Step 4) Repeat Step 2 and Step 3 until the objective function of Problem (16) is less than $\epsilon$, where $\epsilon$ is a small value close to zero.

Inner Loop: Here, we solve the optimization problem under the assumption that the value of $q_{c}$ is given. Therefore, the optimization problem in (16) is a concave problem with one global optimal solution. Hence, we can use the dual problem to achieve the optimal solution, where the strong duality holds in the concave problems. Therefore, using the Lagrangian duality, the dual optimization problem of the problem in (15) can be expressed as follows:

$$
\begin{array}{r}
\zeta=k \sum_{j=1}^{N_{u, c}} \log \left(1+\frac{\left(\rho^{2} / 2\right) P_{j, c}}{B N_{0}+X_{c, j}}\right)-q_{c}^{*} \sum_{i=1}^{N_{A, c}} \sum_{j=1}^{N_{u, c}} g_{i, j}^{2} P_{j, c} \\
+\sum_{j=1}^{N_{A, c}} \mu_{j, c}\left(R_{j, c}-\gamma\right)-\sum_{i=1}^{N_{A, c}} v_{i, c}\left(\sum_{j=1}^{N_{u, c}} g_{i, j}^{2} P_{j, c}-P_{\max }\right),
\end{array}
$$

where $\mu_{j, c} \geq 0, j=1, \ldots, N_{u}$ and $v_{i, c} \geq 0, i=1, \ldots, N_{A, c}$ are the Lagrangian dual variables. From the Karush-KuhnTucker (KKT) conditions [30], we have

$$
\frac{\partial \zeta}{\partial P_{j}}=0
$$

Solving (18), we obtain

$$
P_{j, c}=\frac{\left(1+\mu_{j, c}\right) k}{\ln (2)\left(\sum_{i=1}^{N_{A, c}}\left(v_{i, c}+q\right) g_{i, j}^{2}\right)}-\frac{2\left(N_{0} B+X_{j, c}\right)}{\rho^{2}},
$$

where the variable $\mu_{j, c}$ must be selected to guarantee that the Constraints in (14b) are feasible and $v_{i, c}$ must guarantee that the Constraints in (14c) are feasible too. Now, these dual variables must be found to obtain $P_{j, i} \forall j$. In [31]-[33], the authors used the subgradient method in similar problems to find the optimal dual variable. In brief, the subgradient method gives the dual variable initial values then finds $P_{j, c}$ using 
Equation (19). Then, the dual variables in each iteration can be updated as follows

$$
v_{i, c}(n+1)=v_{i, c}(n)+\delta_{v}\left(\sum_{j=1}^{N_{u, c}} g_{i, j}^{2} P_{j, c}-P_{\max }\right), i=1, \ldots, N_{A, c},
$$

$$
\mu_{j, c}(n+1)=\mu_{j, c}(n)+\delta_{\mu}\left(\gamma-R_{j, c}\right), j=1, \ldots, N_{u, c},
$$

where $\delta_{\mu}$ and $\delta_{v}$ are steps size that should be sufficiently small to guarantee approaching the optimal solution at the steady state. The subgradient is an iterative algorithm that needs a large number of iterations to converge, a very careful selection of the step size, and a very careful selection of the initial values of the dual variables. Therefore, in the following, we find a closed-form expressions for the $\mu^{\prime} s$ dual variables in terms of the $v^{\prime} s$ dual variables. By substituting (19) in Constraint (14b), $\mu_{j, c}$ must be

$$
\begin{array}{r}
\mu_{j, c} \geq \frac{\ln (2)}{k}\left(\frac{P_{j \min } \rho^{2}+2\left(N_{0} B+I_{j, c}\right)}{\rho^{2}}\right) \\
\left(\sum_{i \in N_{A, c}}\left(v_{i, c}+q\right) \mathbf{g}_{j, i}^{2}\right)-1 .
\end{array}
$$

Hence, instead of updating the $\mu^{\prime} s$ dual variables using (21), we update them using (22). We show in details in our previous work [8] that the above substitution increases the rate of convergence significantly with less constraints violation compared to the sub-gradient method.

1) Finding the exact inter-cell interference information: In the literature, the power allocation problem was solved under the assumption that the inter-cell interference is in its maximum value [19]-[21]. The aim of this assumption is to simplify the power allocation problem and to guarantee that the required QoS of the users is achieved. However, this assumption yields a significant degradation in the objective function (EE), since the power is allocated based on inaccurate interference information. Therefore, we provide iterative steps that lead to find the actual interference information and enhance the $\mathrm{EE}$ as we increase the number of iterations. If the complexity is not an issue, we can increase the number of iterations to have a better EE. If not, we should decrease the number of iterations to avoid implementing the power allocation problem several times. The main idea is to implement the power allocation in the different clusters in a successive way and update the inter-cell interference in each step. Thus, the steps are:

1) Implement the power allocation in Cell 1 under the assumption that the interference at the users in that cell is in its maximum value, and then broadcast the allocated transmit powers to all clusters to update their inter-cell interference.

2) Implement Step 1 for Cells $2,3, \ldots, K$ in a consecutive way with updating the interference information in each cell.

3) Repeat Step 2 for all cells (including Cell 1) until no improvement is achieved in the $\mathrm{EE}$, or the number of iterations reaches its limit.
The above steps guarantee improving the EE and achieving the QoS constraints. This is because as we increase the number of iterations, each cell approaches to gather the exact interference information.

\section{Joint Power Allocation and APs Selection}

In the previous section, we provide a solution for allocating the power under a given participating APs. In this section, we first show how the power allocation and APs selection problems are interlinked, then we propose an iterative algorithm to solve both problems jointly. This iterative algorithm can be implemented after clustering the users and associating APs. To prove that the power allocation and the APs selection problems are interlinked, we should show that the allocated powers for the users' messages depend linearly on the transmit power at the APs. From (4), the relation between the transmit powers and the power assigned to the users' messages in cluster $c$ is given by $\mathbf{p}_{c}=\mathbf{G}_{\mathbf{c}}{ }^{2} \mathbf{P}_{c}$, where $\mathbf{p}_{c}$ is the vector power at the APs, and the square at $\mathbf{G}_{c}^{2}$ is the square of the elements in $\mathbf{G}_{c}$. Hence, we can find the powers assigned for the transmitted signals by

$$
\mathbf{P}_{c}=\mathbf{F}_{c} \mathbf{p}_{c},
$$

where $\mathbf{F}_{c}=\left(\mathbf{G}_{\mathbf{c}}{ }^{2 H} \mathbf{G}_{\mathbf{c}}{ }^{2}\right)^{-1} \mathbf{G}_{\mathbf{c}}{ }^{2 H}$. Equation (23) means that switching off any AP (equating its transmit power by zero), affects the allocated power for users directly. Also, adding or inactivating an AP to cluster $c$ affects the signal-to-noise ratio (SNR) of the users, belonging to the other cells, by changing the received interference. Therefore, selecting the participating APs in cell $c$ affects the allocated power in that cell and in the other cells. This motivated us to deal with the power allocation and AP selection problems jointly.

The goal of optimizing the participating APs in communication is to minimize the inter-cell interference. It is clear that, under an optimal power allocation strategy, as we increase the participating APs in the cell $c$, the EE in that cell is improved. On the other hand, these added APs degrade the EE in the other cells by emitting interference. Therefore, first, we classify the APs into three categories: 1) the APs that have zero channels to all users, which must be switched off, 2) the APs that have channels to the associated users but zero channels to the users belonging to other cells (i.e. non-interfering APs), 3) the interfering APs that have LoS to their associated users and also have LoS to the users in the other clusters (i.e. interfering APs). The second category of the APs must be switched on because their participation in the communication improves the $\mathrm{EE}$ as long as the power allocation optimization problem is applied. Our interest is in the third category, where each one of the APs has to be selected carefully to be switched on or switched off. Participating these APs definitely improves the $\mathrm{EE}$ in their own cells, but on the other hand, reduces the EE of the other cells by causing inter-cell interference. Thus, we first establish a metric that approximately measures the eligibility of each AP to participate in the communication (in case it causes non-zero inter-cell interference). This metric of the AP $i$ in the cluster $c$ can be given by

$$
\Upsilon_{i, c}=\frac{\sum_{j \in c} h_{j, i}^{2}}{\sum_{j \notin c} h_{j, i}^{2}} . \forall i \forall c
$$


High value of $\Upsilon_{i, c}$ means that the quality of the information signal coming from the AP $i$ to the intended users is much greater than the emitted interference from that AP and vice versa. The following algorithm solves the power allocation and APs selection alternatively until they converge.

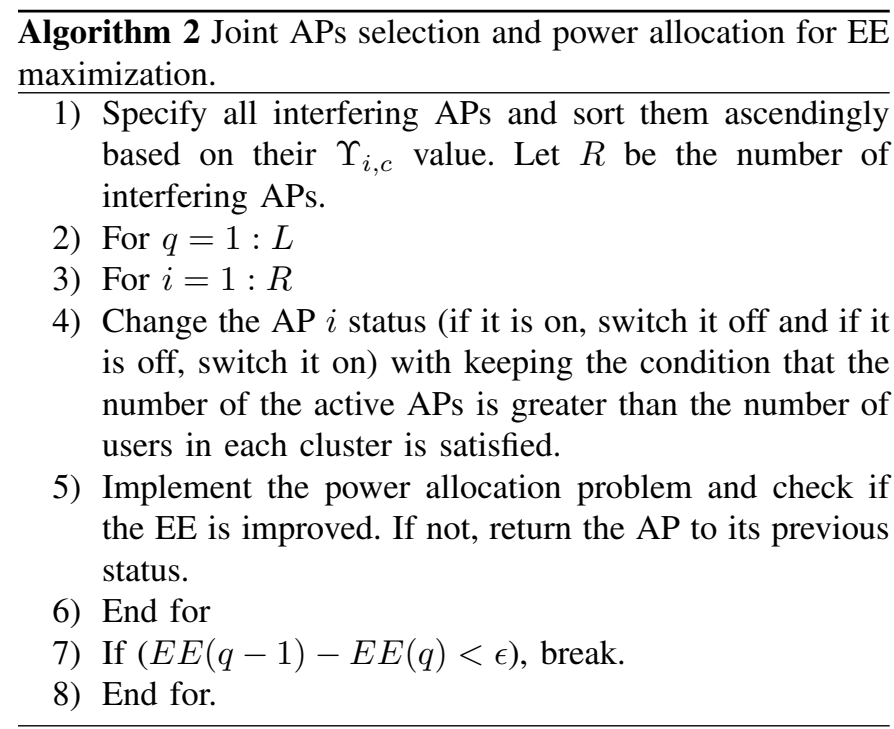

The benefit behind sorting the APs ascendingly based on their $\Upsilon_{i, c}$ values is that we guarantee satisfying the condition that the number of active APs is greater than the number of users in each cluster with the APs that have highest $\Upsilon_{i, c}$. In addition, sorting the APs ascendingly increases the rate of convergence significantly. Condition in Step 4 in Algorithm 2 is needed to guarantee that the intra-cell interference is totally eliminated, since this cannot be guaranteed if the number of APs is less than the number of users. In addition, because of the assumption that the number of users in the system is much less than the number of APs, it is not wise to have some cells crowded with many users (number of users is greater than number of APs in the cell) and some cells have only a few number of users. Therefore, we establish this condition to avoid or alleviate the effect of having unbalanced VLC systems and guarantee zero intra-cell interference.

Algorithm 2 is guaranteed to converge because with each change in the AP status in Step 3, the EE either improves or stays fixed. In addition, it is guaranteed to approach to the optimal solution as we increase the number of iterations. To show that, in each iteration, the proposed algorithm tests all the interfering APs by implementing the power allocation problem with each AP, which means that Algorithm 2 tries all the possible solution to have the best one. The reason to repeat testing the interfering APs is that the optimal state of AP $i$ (on or off) depends on the given states of all other interfering APs, especially the ones that are close to the AP $i$.

1) Suboptimal Approach: Although Algorithm 2 approaches to the optimal joint power allocation and APs selection as we increase the number of iterations, it suffers from high computational complexity. In Algorithm 2, in each AP status change, we need to implement the power allocation problem. In other words, if we have $L$ interfering APs, we need to implement the power allocation problem $L$ times in each iteration. Therefore, in this section, we propose a suboptimal solution for the joint power allocation and APs selection optimization problem that is aimed at decreasing the complexity, by decreasing the number of times of implementing the power allocation problem, and minimizing the degradation in the $\mathrm{EE}$ as much as possible. This algorithm is based on estimating the APs that should be switched off. The main idea of this algorithm is first to implement the power allocation problem when all the APs are participating in communications, then we list all the interfering APs with sorting them ascendingly based on their eligibility factor. After that, we switch off all the APs that are contributing in the EE degradation without implementing the power allocation problem with each AP state change. Then, we can re-implement the power allocation problem after we finish switching off all the harmful APs. It is important to say that when we switch off the AP $i$ in the cell $c$, we need to re-calculate the assigned power for the users in the cluster $c$ using the relation $\mathbf{P}_{c}=\mathbf{F}_{c} \mathbf{p}_{c}$, where the $i^{\text {th }}$ term in $\mathbf{p}_{c}$ equal to zero. Algorithm 3 illustrates the steps to clearly provide a suboptimal solution for the joint power allocation and APs selection optimization problem. In Fig. 4, we show

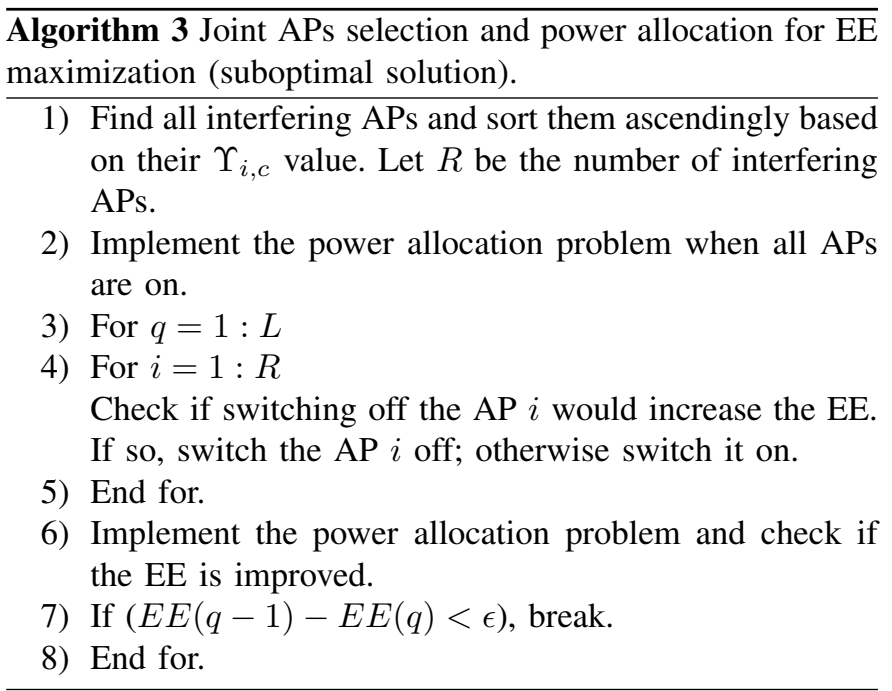

the difference between Algorithms 2 and 3 by applying them in the above example in Fig. 3, and see what are the APs that should be switched off from both algorithms. It seems that both algorithms result in switching off the same APs with two added in Algorithm 3. This is because in Algorithm 3, once it switches off an AP, it does not return to test it again even if switching that AP on would be helpful in increasing the EE.

In the following we show the relationship of the proposed algorithms and steps by presenting a flowchart. Fig. 5 shows the whole proposed process for maximizing the EE based on the UC design. At Step 3 in Fig. 5, we can either use Algorithm 2 or 3 , where the difference between them is that in Algorithm 2 the power allocation optimization problem is implemented with each AP status change, while in Algorithm 3 the power allocation is implemented after testing all the interfering APs.

\section{Simulation Results}

In the following, we verify the capability of the proposed algorithms for enhancing the performance of the VLC net- 


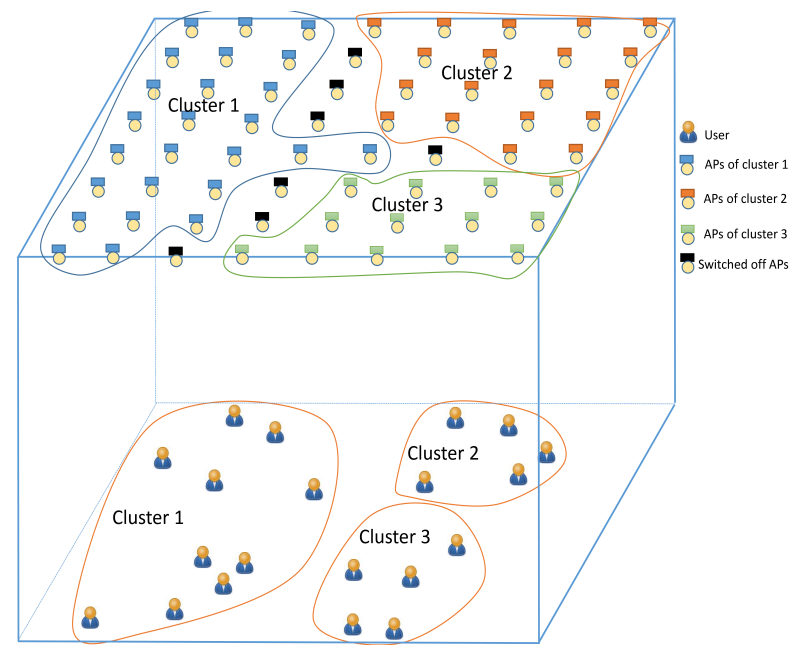

(a) Algorithm 2.

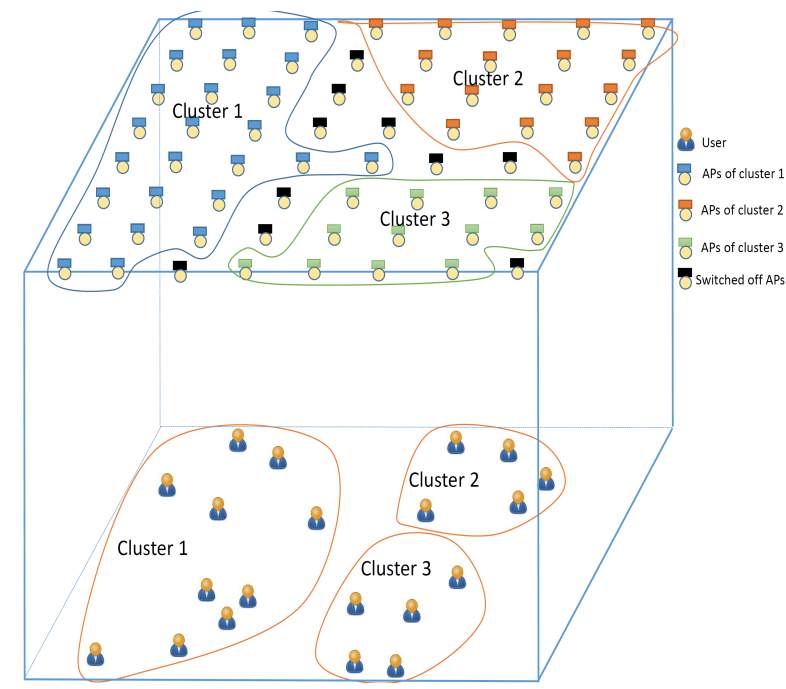

(b) Algorithm 3 .

Fig. 4. An example of switching off APs using a) Algorithm 2, b) Algorithm 3, when $K=3$ and H-FoV $=60^{\circ}$.

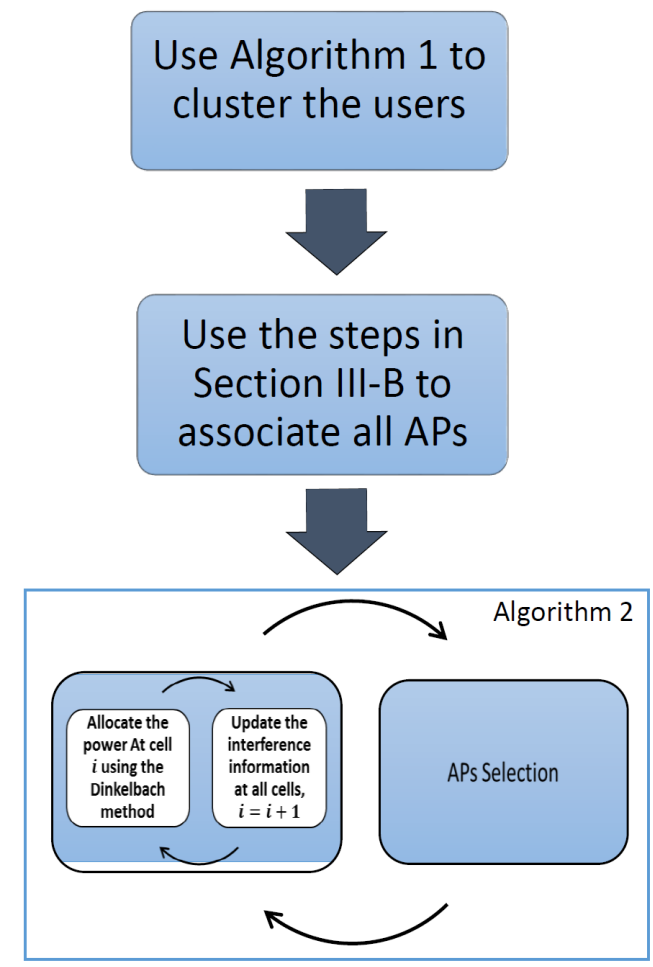

Fig. 5. Flowchart showing how the proposed algorithms and steps would be arranged and implemented to maximize EE.

work. The values of all used parameters in the considered VLC system are given in Table I. Monte-Carlo simulation is used to assess the performance of the proposed algorithms where in each simulation iteration, a uniform random user distribution is generated. We show the convergence of the proposed algorithms and how they improve the system's EE. We compare our work with those in the literature in general such as: 1) in Figures 6 and 10, we compare the approach of allocating the power based on the worst case interference,
TABLE I

\section{SIMULATION PARAMETERS}

\begin{tabular}{lc}
\hline Name of the Parameter & Value of the Parameter \\
\hline Maximum bandwidth of VLC AP, $B$ & $20 \mathrm{MHz}$ \\
The physical area of a PD, $A_{p}$ & $0.1 \mathrm{~cm}^{2}$ \\
Half-intensity radiation angle, $\theta_{1 / 2}$ & $60^{\circ}$ \\
Half FoV (H-FoV) semi-angle of PD, $\Theta$ & $45^{\circ}-65^{\circ}$ \\
Gain of optical filter, $g_{\text {of }}$ & 1 \\
Refractive index, $n$ & 1.5 \\
Efficiency of converting optical to electric, $\rho$ & $0.53[\mathrm{~A} / \mathrm{W}]$ \\
Maximum transmit power [21], $p_{\max }$ & $0.386 \mathrm{Watt}$ \\
Noise power spectral density of LiFi, $N_{0}$ & $10^{-22} \mathrm{~A}^{2} / \mathrm{Hz}$ \\
Room size & $16 \times 16 \mathrm{~m}^{2}$ \\
Room height & $3 \mathrm{~m}$ \\
User height & $0.85 \mathrm{~m}$ \\
Number of APs & $8 \times 8$ \\
Number of users & 20 \\
Monte Carlo & 150 iterations \\
$M$ in the proposed clustering algorithm & 5
\end{tabular}

which is proposed in [20], [21], and [19], and our iterative algorithm that updates the interference information with each iteration, 2) in Figures 8-12, we consider the approach proposed in [20] of participating all APs (that have LoS to any user) in communication as a baseline and compare it with the proposed algorithm for the APs selection, where the approach in [20] is indicated by "without APs selection" in the figures, 3 ) in Fig. 14, we compare the proposed algorithm for APs selection and association with the one proposed in [21], 4) we also compare the proposed clustering method versus the Kmeans++ clustering method presented in [25].

In Fig. 6, we plot the EE versus the number of iterations of updating the interference when all the APs are involved in communications. As can be shown, as the number of iterations increases, the EE increases, and the rate of increasing gets smaller with higher number of iterations. In each iteration, we need to implement the power allocation problem in each cluster which means that increasing the number of iterations would significantly increase the complexity but with a significant enhancement in the performance. Hence, the proposed algorithm for interference updating provides us with 


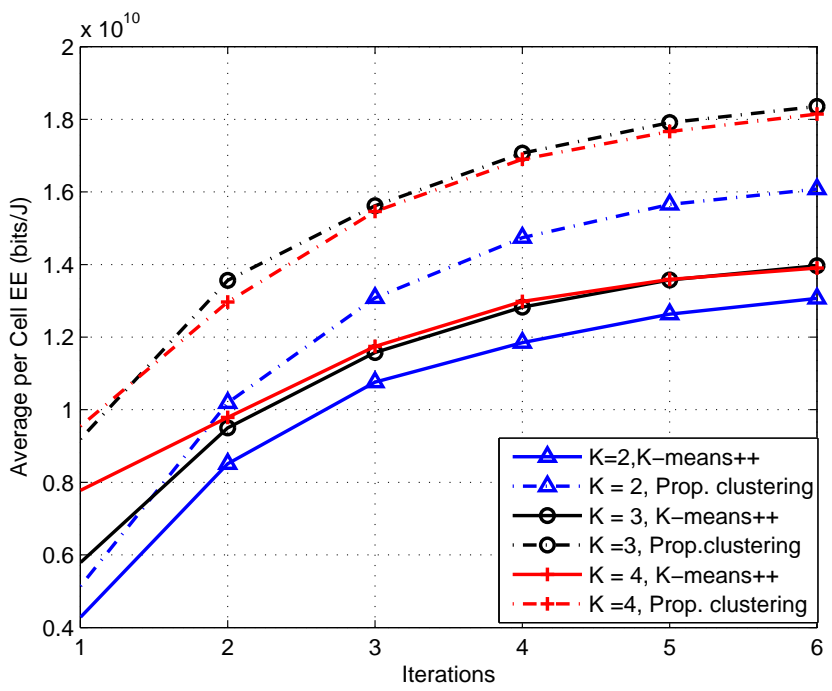

Fig. 6. Energy efficiency versus number of iterations of updating the interference when all the APs are participating, $\mathrm{H}-\mathrm{FoV}=55^{\circ}$.

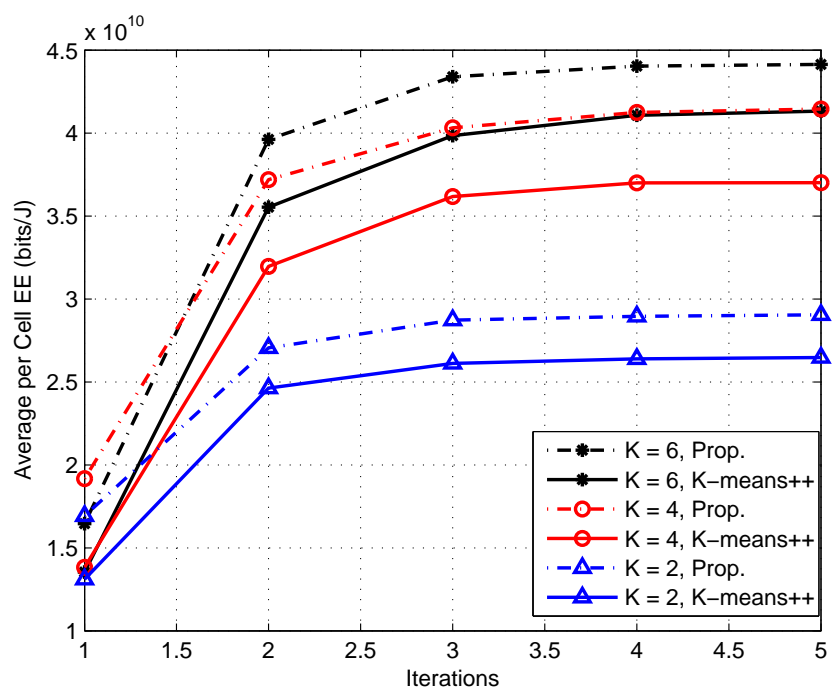

Fig. 7. Energy efficiency versus number of iterations in Algorithm 2 with different number of clusters and different users clustering, $\mathrm{H}-\mathrm{FoV}=55^{\circ}$.

an optional decision whether to enhance the performance at the expense of the complexity or vice versa.

Fig. 7 shows the convergence of Algorithm 2. It is clear that Algorithms 2 needs at most three iterations to converge. Furthermore, the number of iterations needed for convergence does not depend on the number of clusters in the system. In addition, it can be seen that only the first iteration yields a significant improvement in the EE of the system, especially when the number of clusters is large. Furthermore, this figure beside Fig. 6 fairly compare between the user clustering using the K-means++ and the proposed Algorithm 1. Both show the superiority of our proposed users' clustering algorithm over the K-means++ algorithm with different number of clusters whether we implement one iteration or multiple iterations.

Fig. 8 illustrates how the EE behaves as we increase the number of cells. Besides, it shows the significant improve-

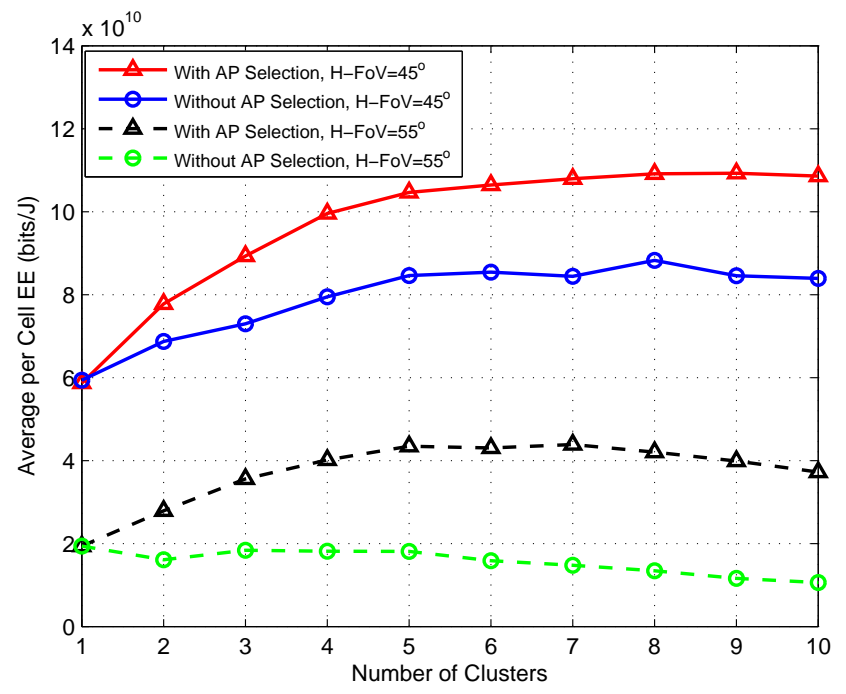

Fig. 8. Comparison between applying Algorithm 2 for AP selection and participating all APs by plotting EE versus number of clusters with different H-FoV.

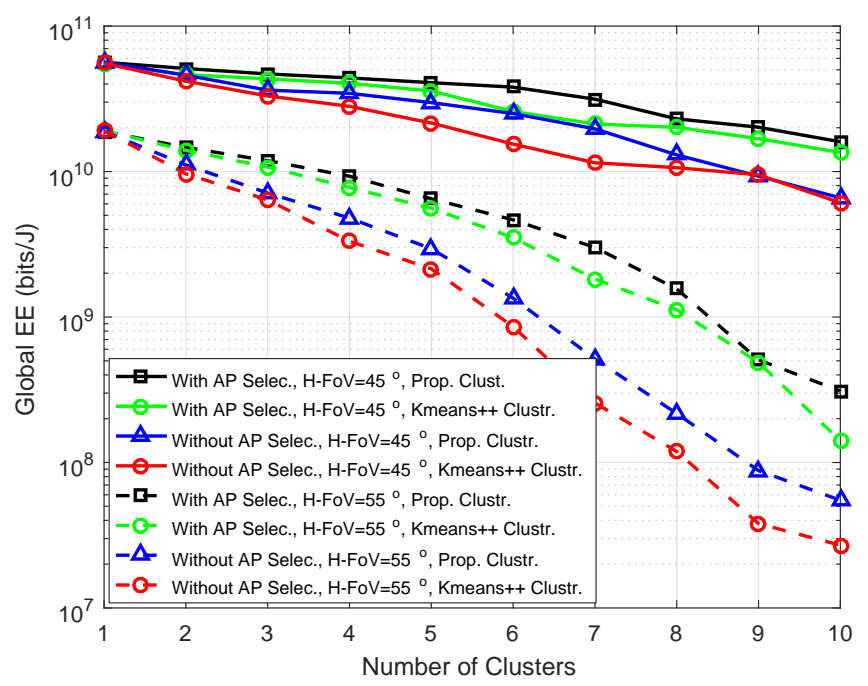

Fig. 9. Comparison between applying Algorithm 2 for AP selection and participating all APs by plotting GEE versus number of clusters with different H-FoV.

ment in the EE when we apply Algorithm 2 compared to participating all the APs in the transmission. This figure shows unexpected results since as we know, as the number of cells increases, the inter-cell interference increases which degrades the EE. But, this figure shows that the average per-cell EE increases and then decreases with the number of cells, which means that there is another factor that helps in enhancing the average per-cell EE as the number of cells increases. This factor is the number of users per cell since as the number of clusters increases the average number of users per cell decreases. This means that the required transmit power per cell decreases as the number of clusters increases, which results in enhancing the average per-cell EE. Apparently, this factor has a great impact when the inter-cell interference is small 


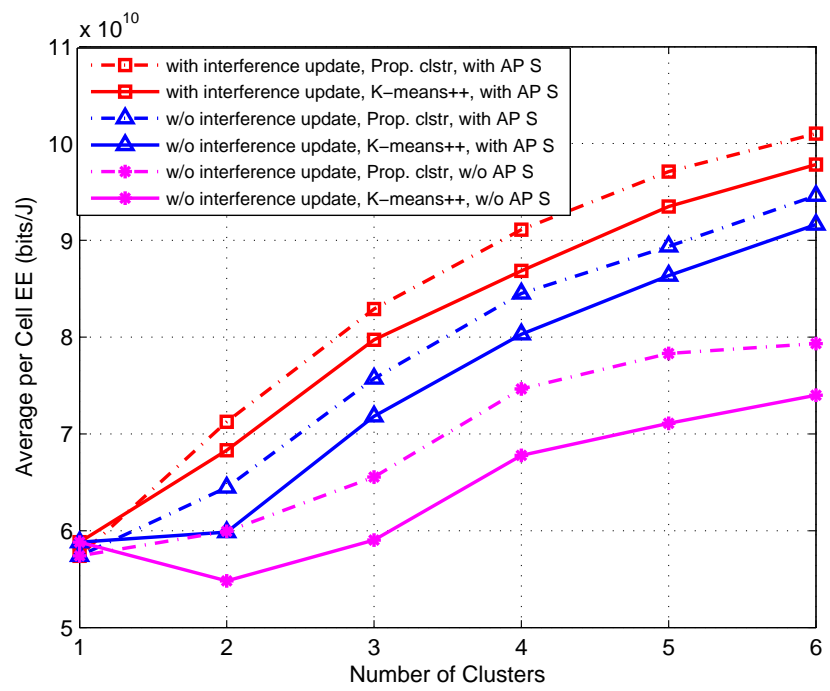

Fig. 10. Comparison between the K-means++ and the proposed clustering methods with and without AP selection by plotting the EE versus number of cells when $\mathrm{H}-\mathrm{FoV}=45^{\circ}$.

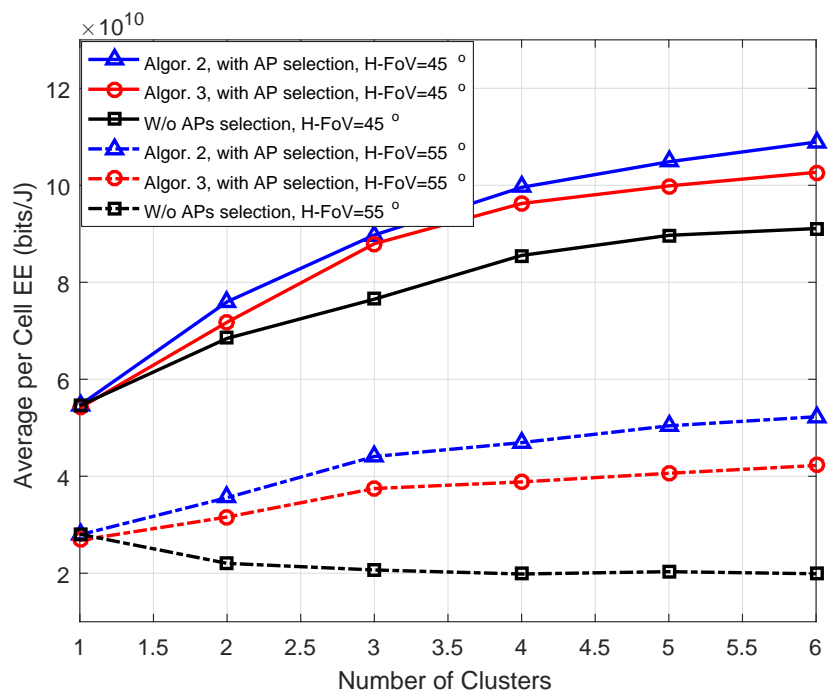

Fig. 11. Comparison between the Algorithm 2 and Algorithm 3 with and without AP selection by plotting the average per cell EE versus number of cells with different H-FoV.

which occurs when the number of clusters is small or when the $\mathrm{H}-\mathrm{FoV}$ is small.

On the other hand, the GEE is decreasing with increasing the number of cells as shown in Fig. 9, because the GEE depends on the number of users in the whole system. It is important to note that in Fig. 9, we implement the proposed algorithms that are established to maximize the average percell $\mathrm{EE}$ and based on the output allocated power, we measure the GEE by calculating Equation (13). Fig. 9 also shows that the proposed user clustering algorithm outperforms the $\mathrm{K}$ means++ algorithm in terms of GEE. Besides, Algorithm 2 provides a significant improvement in GEE as shown in Fig. 9.

Fig. 10, shows the effectiveness of the proposed algorithms in improving the $\mathrm{EE}$ of the considered network. The best

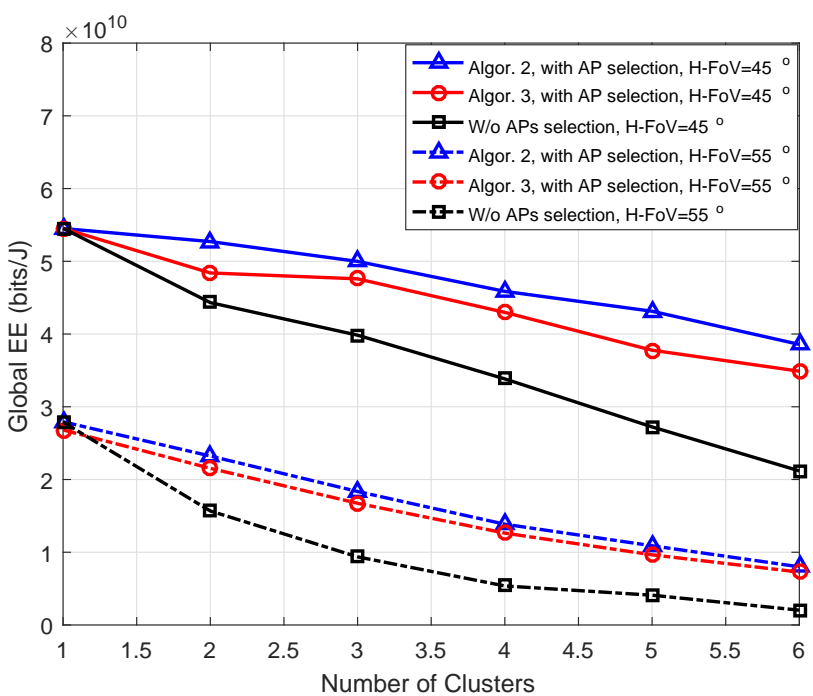

Fig. 12. Comparison between Algorithm 2 and Algorithm 3 with and without AP selection by plotting the GEE versus number of cells with different FoV.

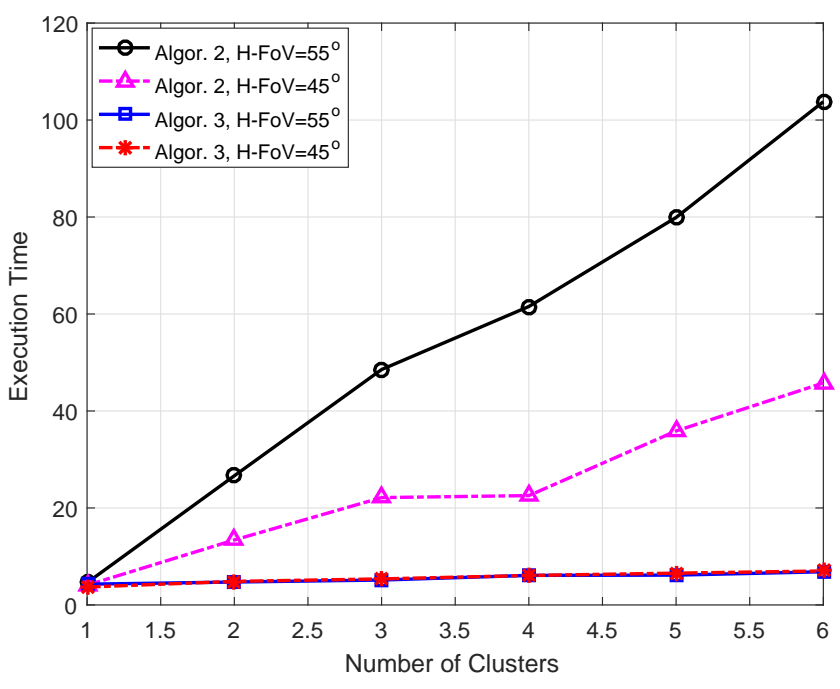

Fig. 13. Comparison between complexity of Algorithms 2 and 3 by plotting the execution time versus number of cells when with different H-FoV.

performance occurs when we apply the AP selection along with the proposed user clustering algorithm with updating the interference, while the worst performance happens when the K-maens++ user clustering algorithm, all the APs participate in communication, and the powers are allocated based on the worst case interference. This figure shows that efficient user clustering methods leads to a significant improvement on the system's EE.

In Figs. 11 and 12, we conduct a fair comparison between Algorithm 2 and Algorithm 3 by plotting the average per cell $\mathrm{EE}$ and the GEE, respectively, versus number of cells. It can be shown that with different number of clusters and different H-FoV, Algorithm 2 is more energy-efficient than Algorithm 3. This is because in Algorithm 2, with each AP status change, we test the average per cell EE by re-implementing the power allocation problem, while in Algorithm 3, we implement the 
power allocation after we test all the interfering APs. In other words, if the number of interfering APs is $L$, in each iteration, we implement the power allocation problem in Algorithm $2 \mathrm{~L}$ times, while we implement the power allocation problem only once in Algorithm 3 with each iteration.

On the other hand, in Fig. 13, we compare both Algorithms 2 and 3 in terms of complexity by plotting the execution time spent by both algorithms. Fig. 13 shows that Algorithm 3 needs much less time than Algorithm 2 to implement the joint power allocation and AP selection. This is because of the reason stated before that in Algorithm 2, with each AP status change, we test the average per cell EE by re-implementing the power allocation problem, while in Algorithm 3, we implement the power allocation after we test all the interfering APs. Because the number of interfering APs is increased as we increase $\mathrm{H}-\mathrm{FoV}$ or the number of clusters, Fig. 13 shows that we need much time to test all the interfering APs and select the APs that must participate in communication.

In Fig. 14, we compare our algorithms for APs association with the algorithm that was proposed in [21]. The comparison is conducted under the same user clustering algorithm, the same power allocation algorithm, and with 200 Monte-Carlo simulations. The difference between them is that we deal with the power allocation problem and AP association jointly, while they solved both problems separately. In addition, we establish a metric for each AP based on its average channels to the clustered users aiming to select the best cell to serve and to mitigate the inter-cell interference. The algorithm proposed in [21] can be briefly explained by that, after selecting the anchor AP for each user, the rest of the APs are associated to the clusters within a certain range $d_{\alpha}$. In other words, the AP $i$ is assigned to the cluster $c$ if the distance between that AP and the center of that cluster is less than or equal to $d_{\alpha}$. If more than one cluster satisfy this condition for AP $i$, this AP must be switched off to mitigate the interference. The disadvantages of this approach are: increasing or decreasing $d_{\alpha}$ may switch on the harmful APs and switch off the helpful ones because it depends only on the distance and not on the channel that is affected by different factors such as the receiver FoV, the transmitter coverage and so on; switching the APs off or on is not related to the transmit power at these APs, which is a crucial factor to consider. Therefore, as shown in Fig. 14, our proposed algorithms (Algorithms 2 and 3) significantly outperform the proposed algorithm in [21] for AP association in terms of the average per cell EE.

In Fig. 15, we show how the average per cell EE behaves when we change the number of users in the system with different H-FoV. As shown in this figure, the EE is degraded as we increase the number of users. This is because the amount of required power needed by users to achieve the required QoS increases as we increase the number of users. It is also shown that the amount of improvement in the EE caused by APs selection increases as we increase the number of users. This is because the number of interfering APs increases with higher number of users, which means that the AP selection is highly demanded when we have a high number of interfering APs. In other words, the AP selection algorithms have a slight impact on the EE if we have 10 users (small number of interfering

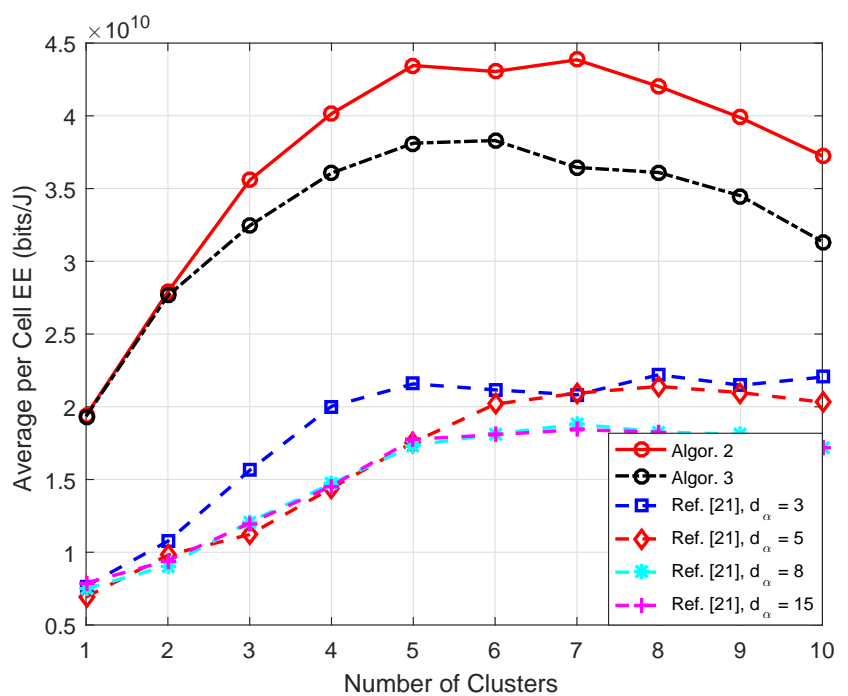

Fig. 14. Comparison of our proposed algorithms (Algorithms 2 and 3) to the Algorithm proposed in [21] in terms of APs association to the clustered users, $\mathrm{H}-\mathrm{FoV}=55^{\circ}$

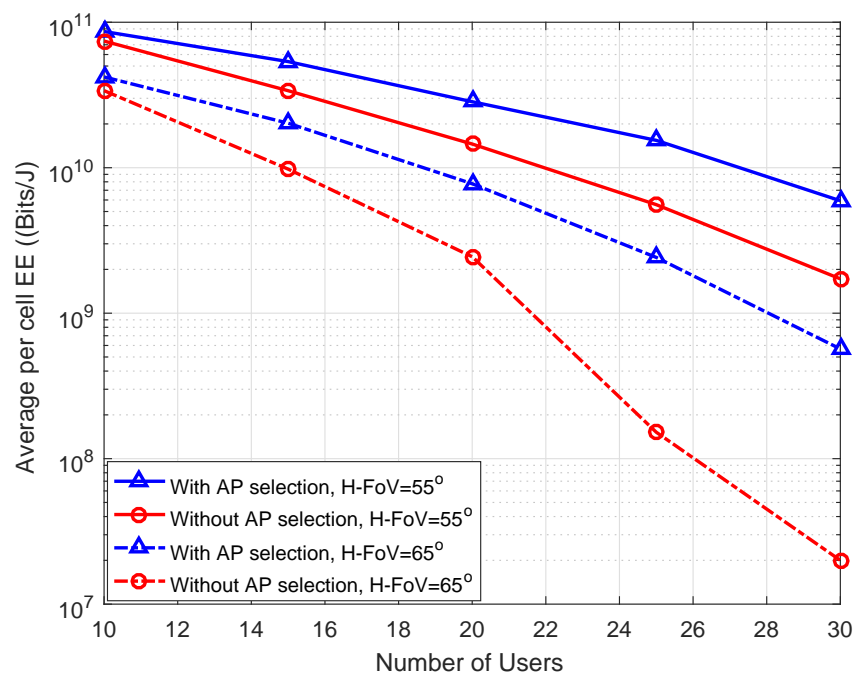

Fig. 15. Energy efficiency versus the number of users in the system with different H-FoV when we have 5 clusters, Algorithm 3 is used for AP selection.

APs), while the impact on the EE would be significant when we have 30 users (large number of interfering APs).

\section{CONCLUSION AND Future WORKS}

In this paper, an energy-efficient VLC network was designed based on amorphous cell formation with jointly allocating the power and associating the APs. A new user clustering algorithm was proposed based on distances between users and separating the clusters as much as possible to mitigate the inter-cell interference. Also, two other algorithms were proposed to jointly allocate the power and select the APs in order to maximize the EE. The aim of the first algorithm is to seek for the optimality of the EE maximization problem, while the aim of the second algorithm was to decrease the 
complexity of the first one with an acceptable degradation in the EE. We also proposed an iterative algorithm to find the optimal power allocation based on exact interference information, which significantly improved the EE. The results showed that designing an appropriate user clustering algorithm with considering the inter-cell interference, selecting the active APs based on the allocated power, and allocating the power based on the exact inter-cell interference information results a significant improvement in EE maximization problem.

As a future work, we recommend to apply the UC design in the hybrid RF/VLC networks, where the edge-users and the mobile users can be connected to RF APs to mitigate the inter-cell interference and the handover overhead, respectively. In addition, we recommend to formulate the optimization problem in terms of maximizing the GEE by introducing a central controller that can gather the information from all cells and then implement the power allocation problem.

\section{ACKNOWLEDGMENT}

This work was funded by the Deanship of Scientific Research at the King Fahd University of Petroleum \& Minerals (KFUPM) through grant number KAUST004. The work was also supported by the National Plan for Science, Technology, and Innovation (Maarifah)-King Abdulaziz City for Science and Technology-through the Science and Technology Unit at KFUPMthe Kingdom of Saudi Arabia, under grant number 15-ELE4157-04. The authors would also like to acknowledge the KFUPM-KAUST research initiative that resulted from this research work.

\section{REFERENCES}

[1] M. Obeed, A. M. Salhab, S. A. Zummo, and M.-S. Alouini, "Joint power allocation and cell formation for energy-efficient VLC networks," in Proc. IEEE International Conf. on Commun. (ICC), Kansas, USA, 2018.

[2] C. Chen, S. Videv, D. Tsonev, and H. Haas, "Fractional frequency reuse in dco-ofdm-based optical attocell networks," J. Lightw. Technol., vol. 33, no. 19, pp. 3986-4000, 2015.

[3] S. Buzzi, I. Chih-Lin, T. E. Klein, H. V. Poor, C. Yang, and A. Zappone, "A survey of energy-efficient techniques for $5 \mathrm{G}$ networks and challenges ahead," IEEE J. Sel. Areas Commun., vol. 34, no. 4, pp. 697-709, 2016.

[4] M. Kavehrad, "Sustainable energy-efficient wireless applications using light," IEEE Commun. Mag., vol. 48, no. 12, pp. 66-73, 2010.

[5] A. Yesilkaya, E. Basar, F. Miramirkhani, E. Panayirci, M. Uysal, and H. Haas, "Optical MIMO-OFDM with generalized LED index modulation,” IEEE Trans. Commun., vol. 65, no. 8, pp. 2429-3441, 2017.

[6] J. Armstrong, "OFDM for optical communications," IEEE/OSA J. Lightw. Technol., vol. 27, no. 3, pp. 189-204, 2009.

[7] X. Li, R. Zhang, and L. Hanzo, "Cooperative load balancing in hybrid visible light communications and WiFi," IEEE Trans. Commun., vol. 63, no. 4, pp. 1319-1329, 2015.

[8] M. Obeed, A. M. Salhab, S. A. Zummo, and M.-S. Alouini, "Joint optimization of power allocation and load balancing for hybrid vlc/rf networks," IEEE/OSA J. Opt. Commun. and Netw., vol. 10, no. 5, pp. 553-562, 2018.

[9] Y. Wang, D. A. Basnayaka, and H. Haas, "Dynamic load balancing for hybrid $\mathrm{Li}-\mathrm{Fi}$ and RF indoor networks," in Proc. IEEE Int. Conf. Commun. Workshops (ICCW), London, UK, 2015, pp. 1422-1427.

[10] K. Chitti, Q. Kuang, and J. Speidel, "Joint base station association and power allocation for uplink sum-rate maximization," in Signal Processing Advances in Wireless Communications (SPAWC), 2013 IEEE 14th Workshop on. IEEE, 2013, pp. 6-10.

[11] M. Obeed, A. M. Salhab, S. A. Zummo, and M.-S. Alouini, "Joint load balancing and power allocation for hybrid VLC/RF networks," in Proc. IEEE Global Commun. Conf. (Globecom), Singapore, 2017.
[12] R. Jiang, Q. Wang, H. Haas, and Z. Wang, "Joint user association and power allocation for cell-free visible light communication networks," IEEE J. Sel. Areas Commun., vol. 36, no. 1, pp. 136-148, 2018.

[13] H. Shen, Y. Deng, W. Xu, and C. Zhao, "Rate-maximized zero-forcing beamforming for vlc multiuser miso downlinks," IEEE Photon. J., vol. 8, no. 1, pp. 1-13, 2016.

[14] A. Nuwanpriya, S.-W. Ho, and C. S. Chen, "Indoor MIMO visible light communications: Novel angle diversity receivers for mobile users," IEEE J. Sel. Areas Commun., vol. 33, no. 9, pp. 1780-1792, 2015.

[15] C. Chen, D. Tsonev, and H. Haas, "Joint transmission in indoor visible light communication downlink cellular networks," in Globecom Workshops (GC Wkshps), 2013 IEEE. IEEE, 2013, pp. 1127-1132.

[16] L. Chen, W. Wang, and C. Zhang, "Coalition formation for interference management in visible light communication networks," IEEE Trans. veh. technol., vol. 66, no. 8, pp. 7278-7285, 2017.

[17] C. Chen and H. Haas, "Performance evaluation of downlink cooperative multipoint joint transmission in lifi systems," in Globecom Workshops (GC Wkshps), 2017 IEEE. IEEE, 2017, pp. 1-6.

[18] R. Zhang, J. Wang, Z. Wang, Z. Xu, C. Zhao, and L. Hanzo, "Visible light communications in heterogeneous networks: Paving the way for user-centric design," IEEE Wireless Commun., vol. 22, no. 2, pp. 8-16, 2015.

[19] X. Li, F. Jin, R. Zhang, J. Wang, Z. Xu, and L. Hanzo, "Users first: User-centric cluster formation for interference-mitigation in visible-light networks," IEEE Trans. Wireless Commun., vol. 15, no. 1, pp. 39-53, 2016.

[20] R. Zhang, H. Claussen, H. Haas, and L. Hanzo, "Energy efficient visible light communications relying on amorphous cells," IEEE J. Sel. Areas Commun., vol. 34, no. 4, pp. 894-906, 2016.

[21] X. Li, Y. Huo, R. Zhang, and L. Hanzo, "User-centric visible light communications for energy-efficient scalable video streaming," IEEE Trans. Green Commun. Netw., vol. 1, no. 1, pp. 59-73, 2017.

[22] Y. Wang and H. Haas, "Dynamic load balancing with handover in hybrid Li-Fi and Wi-Fi networks," IEEE/OSA J. Lightw. Technol., vol. 33, no. 22, pp. 4671-4682, Nov. 2015.

[23] T. Komine and M. Nakagawa, "Fundamental analysis for visible-light communication system using led lights," IEEE Trans. Consum. Electron., vol. 50, no. 1, pp. 100-107, 2004.

[24] J. M. Kahn and J. R. Barry, "Wireless infrared communications," Proc. IEEE, vol. 85, no. 2, pp. 265-298, 1997.

[25] D. Arthur and S. Vassilvitskii, "K-means++: The advantages of careful seeding," in Proc. The 8th Annual ACM-SIAM Sympo. Discrete Algorithms. Society for Industrial and Applied Mathematics, 2007, pp. 1027-1035.

[26] S. Feng, X. Li, R. Zhang, M. Jiang, and L. Hanzo, "Hybrid positioning aided amorphous-cell assisted user-centric visible light downlink techniques," IEEE Access, vol. 4, pp. 2705-2713, 2016.

[27] A. Zappone, E. Jorswieck et al., "Energy efficiency in wireless networks

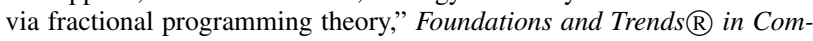
munications and Information Theory, vol. 11, no. 3-4, pp. 185-396, 2015.

[28] A. Zappone, L. Sanguinetti, G. Bacci, E. Jorswieck, and M. Debbah, "Energy-efficient power control: A look at $5 \mathrm{~g}$ wireless technologies," IEEE Trans. Signal Process., vol. 64, no. 7, pp. 1668-1683, 2016.

[29] W. Dinkelbach, "On nonlinear fractional programming," Management Science, vol. 13, no. 7, pp. 492-498, 1967.

[30] S. Boyd and L. Vandenberghe, Convex Optimization. Cambridge University Press, 2004.

[31] M. Kashef, M. Ismail, M. Abdallah, K. A. Qaraqe, and E. Serpedin, "Energy efficient resource allocation for mixed RF/VLC heterogeneous wireless networks," IEEE J. Sel. Areas Commun., vol. 34, no. 4, pp. 883-893, 2016

[32] H. Wang, A. Liu, and X. Pan, "Optimization of joint power and bandwidth allocation in multi-spot-beam satellite communication systems," Math. Problems in Eng., vol. 2014, 2014.

[33] D. W. K. Ng, E. S. Lo, and R. Schober, "Energy-efficient resource allocation in multi-cell ofdma systems with limited backhaul capacity," IEEE Trans. Wireless Commun., vol. 11, no. 10, pp. 3618-3631, 2012. 


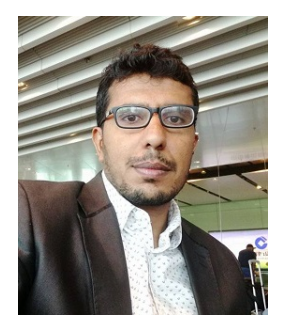

Mohanad Obeed (S'17) received the B.Eng. degree in computer and communication engineering from Taiz University, Taiz, Yemen, in 2008, the M.Sc. degree in electrical engineering from King Fahd University of Petroleum and Minerals (KFUPM), Dhahran, Saudi Arabia, in 2016.

$\mathrm{He}$ is currently pursuing the Ph.D. degree at KFUPM, Dhahran, Saudi Arabia. His research interests include visible light communications, cooperative communication, resource allocation, convex optimization, physical layer security, and energy

harvesting.

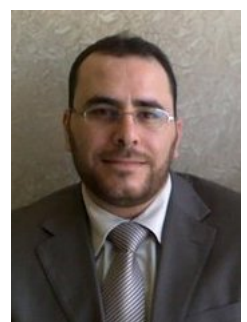

Anas M. Salhab (S'11-M'14-SM'17) received the B.Sc. degree in electrical engineering from Palestine Polytechnic University, Hebron, Palestine, in 2004, the M.Sc. degree in electrical engineering from Jordan University of Science and Technology, Irbid, Jordan, in 2007, and the Ph.D. degree from King Fahd University of Petroleum and Minerals (KFUPM), Dhahran, Saudi Arabia, in 2013. From 2013 to 2014, he was a Postdoctoral Fellow with the Electrical Engineering Department, KFUPM. He is currently an Assistant Professor and the Assistant Director of the Science and Technology Unit with the Deanship of Scientific Research, KFUPM. His research interest spans special topics in modeling and performance analysis of wireless communication systems, including cooperative relay networks, cognitive radio relay networks, free space optical networks, visible light communications, and co-channel interference. He was selected as an Exemplary Reviewer by the IEEE WIRELESS COMMUNICATIONS LETTERS for his reviewing service in 2014. Recently, he received the KFUPM Best Research Project Award as a Co-investigator among the projects in 2013/2014 and 2014/2015.

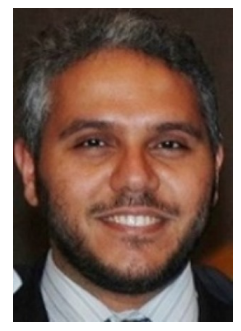

Salam A. Zummo (M'00-SM'08) received the B.Sc. and M.Sc. degrees in electrical engineering from the King Fahd University of Petroleum and Minerals (KFUPM), Dhahran, Saudi Arabia, in 1998 and 1999, respectively, and the Ph.D. degree from the University of Michigan, Ann Arbor, USA, in 2003. He is currently a Professor with the Electrical Engineering Department, KFUPM. Prof. Zummo has six issued U.S. patents and authored over 100 papers in reputable journals and conference proceedings. His research interests are in the area of wireless communications, including cooperative diversity, cognitive radio, multiuser diversity, scheduling, MIMO systems, error control coding, multihop networks, and interference modeling and analysis in wireless systems. He received the Saudi Ambassador Award for early Ph.D. completion in 2003, and the British Council/BAE Research Fellowship Awards in 2004 and 2006. He also received the KFUPM Excellence in Research Award from 2011 to 2012.

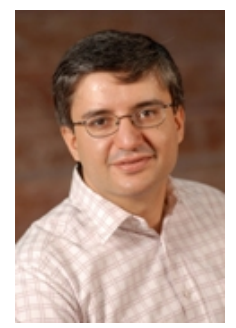

Mohamed-Slim Alouini (S'94, M'98, SM'03, F'09) was born in Tunis, Tunisia. He received the Ph.D. degree in Electrical Engineering from the California Institute of Technology (Caltech), Pasadena, CA, USA, in 1998. He served as a faculty member in the University of Minnesota, Minneapolis, MN, USA, then in the Texas A\&M University at Qatar, Education City, Doha, Qatar before joining King Abdullah University of Science and Technology (KAUST), Thuwal, Makkah Province, Saudi Arabia as a Professor of Electrical Engineering in 2009.

His current research interests include the modeling, design, and performance analysis of wireless communication systems. 